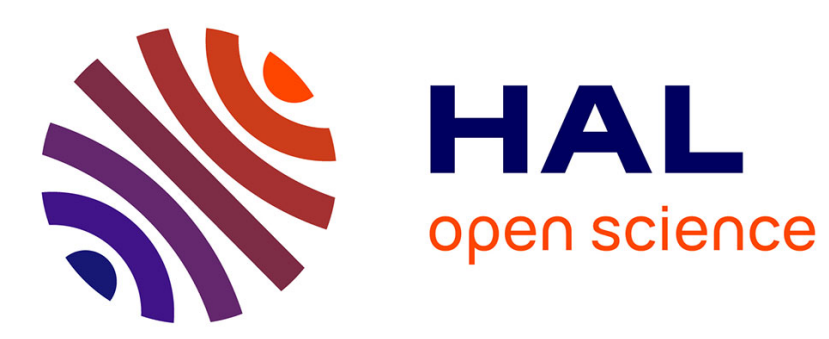

\title{
Les centres éducatifs fermés : de la clôture institutionnelle à l'espace éducatif
}

Catherine Lenzi, Philip Milburn

\section{To cite this version:}

Catherine Lenzi, Philip Milburn. Les centres éducatifs fermés : de la clôture institutionnelle à l'espace éducatif. Espaces et sociétés (Paris, France), 2015, 162 (3), 10.3917/esp.162.0095 hal-01651427

\section{HAL Id: hal-01651427 \\ https://hal.univ-rennes2.fr/hal-01651427}

Submitted on 29 Nov 2017

HAL is a multi-disciplinary open access archive for the deposit and dissemination of scientific research documents, whether they are published or not. The documents may come from teaching and research institutions in France or abroad, or from public or private research centers.
L'archive ouverte pluridisciplinaire HAL, est destinée au dépôt et à la diffusion de documents scientifiques de niveau recherche, publiés ou non, émanant des établissements d'enseignement et de recherche français ou étrangers, des laboratoires publics ou privés. 


\section{LES CENTRES ÉDUCATIFS FERMÉS : DE LA CLÔTURE INSTITUTIONNELLE À L'ESPACE ÉDUCATIF}

Catherine Lenzi, Philip Milburn

\section{ERES | « Espaces et sociétés »}

2015/3 n 162 | pages 95 à 110

ISSN 0014-0481

ISBN 9782749248219

Article disponible en ligne à l'adresse :

https://www.cairn.info/revue-espaces-et-societes-2015-3-page-95.htm

\section{Pour citer cet article :}

Catherine Lenzi, Philip Milburn« Les centres éducatifs fermés : de la clôture institutionnelle à l'espace éducatif », Espaces et sociétés 2015/3 (n 162), p. 95-110. DOI 10.3917/esp.162.0095

Distribution électronique Cairn.info pour ERES.

(C) ERES. Tous droits réservés pour tous pays.

La reproduction ou représentation de cet article, notamment par photocopie, n'est autorisée que dans les limites des conditions générales d'utilisation du site ou, le cas échéant, des conditions générales de la licence souscrite par votre établissement. Toute autre reproduction ou représentation, en tout ou partie, sous quelque forme et de quelque manière que ce soit, est interdite sauf accord préalable et écrit de l'éditeur, en dehors des cas prévus par la législation en vigueur en France. Il est précisé que son stockage dans une base de données est également interdit. 


\title{
Les centres éducatifs fermés : de la clôture institutionnelle à l'espace éducatif
}

\section{Catherine Lenzi Philip Milburn}

\begin{abstract}
Les centres éducatifs fermés (CEF) ont été créés par la loi du 9 septembre 2002 pour répondre aux exigences sécuritaires de la période politique et pour fournir un cadre de « sanction éducative » selon ses termes, à des mineurs qui commettent des infractions de manière répétitive et dont les prises en charge éducatives classiques - en milieu familial ou placé en établissement éducatif - n'ont pas permis d'infléchir le comportement. Plusieurs établissements ont été créés au fil des années et il existe aujourd'hui une soixantaine de centres en activité, dont une large majorité relève d'associations privées habilitées par la Protection judiciaire de la jeunesse ${ }^{1}$ et non directement de ses services.
\end{abstract}

Catherine Lenzi, responsable de la recherche et du laboratoire ESPASS à l'IREIS Rhône-Alpes et chercheur au laboratoire PrinTemps/CNRS catherine.lenzi@gmail.com

Philip Milburn, professeur à l'Université Rennes 2 et chercheur au laboratoire ESO-UMR 6590 milburn@uhb.fr

1. PJJ : administration du ministère de la Justice qui a en charge les mineurs placés sous main de justice. 
Le texte de loi initial prévoit également la création d'Établissements pénitentiaires pour mineurs (EPM), véritables prisons dédiées aux mineurs et incorporant des services éducatifs et scolaires dont six ont été mis en service depuis 2006, les mineurs pouvant être incarcérés dans ce cadre lorsque le tribunal l'estime nécessaire.

Parce que les CEF ne sont ni des prisons (contrairement aux EPM créés dans la même période) ni de simples foyers éducatifs, ils constituent plutôt un espace d'enfermement hybride destiné aux mineurs placés pour faits délictueux, soumis à la double contrainte de la restriction de leurs libertés dans le présent et de l'obligation de participation à des activités pour préparer leur avenir ${ }^{2}$. Notre propos ici consiste dès lors à interroger la manière dont différents acteurs des CEF, mineurs placés et membres du personnel, s'approprient et intègrent le cadre spatial de la contrainte, et dans quelle mesure ils dégagent par ailleurs des espaces affranchis de ces contraintes, tels que Erving Goffman les identifiait dans son œuvre magistrale sur l'asile psychiatrique (Goffman, 1968).

Les réflexions qui traversent le présent article résultent de plusieurs programmes de recherches consacrés à ce type d'établissements (EPM et CEF) dont un en cours sur une série de six $\mathrm{CEF}^{3}$. Il s'agit notamment pour ce dernier, de monographies complètes sous la forme d'une immersion dans la vie des centres effectuée par trois binômes de chercheurs, sur le mode ethnographique, avec des entretiens auprès des personnels (de direction et éducatif) et une exploitation des documents institutionnels. Les données y ont été recueillies de manière régulière au rythme d'une immersion d'environ deux jours par mois durant sept mois. Une grille de collecte des données a été établie de manière équivalente pour chaque établissement.

Les éléments livrés ici sont extraits des résultats empiriques de ce travail, mais n'en constituent qu'un aspect limité et spécifique, consacré à la question de la force institutionnelle du rapport instauré avec l'espace, ses frontières et

2. Alors que les prisons sont gérées par l'administration pénitentiaire et accueillent des mineurs condamnés à une peine, les CEF restent des établissements sociaux contrôlés par la Protection judiciaire de la jeunesse. Pour une comparaison entre CE, EPM et quartiers pour mineurs en maisons d'arrêt : Bailleau et al., 2012.

3. Cf. Bailleau et al., 2012, et « La construction des compétences éducatives, managériales et collectives en milieux contraints », sous la dir. de C. Lenzi et Ph. Milburn, ESPASS-ERP (programme en cours 2012-2014). Cette deuxième recherche a mobilisé dans sa réalisation (collecte, analyse des données et production des rapports), une équipe de neuf personnes, dont les responsables scientifiques du programme, cinq formateurs-chercheurs, un doctorant et un acteur du champ professionnel (Léo Farcy-Callon, Yves Darnaud, Daniel Lepecq, Frédérique Mazereau, Sandrine Sanchez, Nordine Touil, Luc Trouiller). Les deux programmes ont été financés par la mission de recherche « Droit et justice» (ministère de la Justice et CNRS). Ces programmes de recherche comportent d'autres dimensions que celles présentées dans le présent article. 
les configurations qui en résultent. Cet aspect constitue par définition un aspect majeur de la fonction institutionnelle fondée sur le maintien des mineurs dans un espace circonscrit et dans une série d'espaces intérieurs (chambre, cour, salle de classe, jardin, etc.) ou hors de certains espaces. Mieux, compte tenu du temps passé dans les centres (séjour de six mois prévu par les textes), le rapport à l'espace acquiert une dimension de socialisation majeure, au sens de l'apprentissage de la vie sociale, donc éducative, mais pas nécessairement dans le sens visé par l'institution (Dubar, 2002).

Le traitement de la dimension socio-spatiale développée au fil de cet article repose principalement sur l'approche ethnographique des programmes de recherche, notamment l'observation des occupations de l'espace, des circulations et des exploitations des espaces par les différents acteurs présents, mais aussi des objets qui produisent du sens spatial pour les acteurs qui les réinterprètent. Elle est corroborée par les propos tenus en entretiens relatifs à ces questions qui permettent de saisir la portée symbolique que donnent les acteurs, notamment professionnels, à cette dimension de spatialité contrôlée, ainsi qu'aux intentions institutionnelles présentes dans les documents officiels (projet d'établissement, règlement, etc.) et dans les propos des représentants de l'institution ${ }^{4}$.

Accueillant entre huit et douze mineurs, la plupart des CEF sont implantés dans des espaces ruraux, éloignés des habitations, pour des raisons multiples : le coût est une raison triviale mais qui prévaut certainement, il faut aussi éviter l'inquiétude du voisinage ; des raisons à visée éducative ont été envisagées par les promoteurs telles que les dépayser vis-à-vis de leur cadre de vie habituel, souvent très urbain, et de ses présumées influences de socialisation à la déviance. Quelle que soit son intention, l'isolement des CEF constitue le premier élément de clôture dans la mesure où il pose une distance entre la résidence des mineurs placés et leur environnement familier, urbain le plus souvent.

En général, les centres occupent des locaux déjà existants et réaménagés. Ils comportent une cour centrale qui délimite des bâtiments différents et dédiés, où les mineurs placés ont des accès contrôlés : chambres, salles de jeu, cantine, salle de classe, bureaux administratifs, espaces verts (pour des activités horticoles), parfois terrain de sport et gymnase. L'ensemble est délimité par des murs, quelquefois des grillages, jamais de barbelés ou de barreaux; il existe rarement de caméras de surveillance mais toujours des portails automatiques pour l'accès. Ces deux éléments, comme parfois d'autres, viennent symboliser

4. Compte tenu du volume limité de l'article, nous ne pouvons livrer toutes les données pertinentes à cet égard ni présenter la mise en perspective croisée qui a été effectuée dans l'opération d'analyse scientifique. 
la clôture davantage que des murs ou des barrières, l'idée étant d'en faire un lieu de vie plutôt qu'un espace d'enfermement.

Aussi peut-on être surpris, lors de la visite du CEF de Saint-Pamard ${ }^{5}$, d'arriver dans un lieu qui ressemble davantage, par de multiples aspects, à une maison, un lieu de vie familial, qu'à un lieu de détention. Point de clôtures hautes, aucun système de surveillance. Une allée d'arbres et une palissade de canisses forment un paravent qui sépare le lieu de résidence, du chemin de terre qui longe les abords du CEF. Un jardin joliment entretenu offre un espace accueillant, qui ouvre sur une cour centrale où sont disposées deux grandes tables en bois pour les jeunes placés. Lors de nos allées et venues au CEF, il n'est pas rare de trouver quelquesuns d'entre eux errant le long du parking extérieur, regroupés autour d'une voiture, attendant qu'un éducateur les rejoigne pour les conduire à leurs activités extérieures.

Pour autant, bien que l'impression d'un mouvement ouvert imprègne les lieux de toutes parts et permette de saisir sur le vif le bouillonnement d'un quotidien où les mineurs vont et viennent sans répit, de la cour aux espaces de vie commune (salle de télévision, réfectoire et cuisine...), il n'en demeure pas moins qu'une coupure entre deux mondes - «ouvert» et «fermé »apparaît très vite. Aussi nous employons nous dans un premier temps à mettre en évidence ce qui fait fermeture au sein d'un CEF.

\section{DE LA FERMETURE À L'EMPRISE}

Plus encore que la prison contemporaine, de plus en plus tournée vers le monde extérieur (semi-liberté, libération conditionnelle, surveillance électronique, $c f$. Rostaing, 1997 ; Combessie, 2000 ; Chantraine, 2000), les CEF autorisent une circulation régulière entre le dedans et le dehors et ne sauraient être considérés comme un monde parfaitement clos. Ils interrogent de ce fait les usages heuristiques du concept d'institution totale tel que posé par Goffman. Si cette forme de détotalisation du monde carcéral induite par la politique d' « humanisation» et de «responsabilisation» accroît les marges de manœuvres des détenus et évite un délitement identitaire des reclus propre à l'institution totale (Chantraine, op. cit.), elle ne rend pas pour autant caduque la thèse goffmanienne analysant les espaces d'enfermement comme des espaces contraignants et dépersonnalisants. "En effet, Goffman soulignait déjà que l'assujettissement du détenu est assuré non seulement par la surveillance et la discipline, mais aussi par les limites mises à la contrainte exercée par l'institution qui, pour sa propre survie, est obligée de susciter une certaine participation de sa population : assurer un minimum de bien-être, développer un

5. Les noms des établissements cités sont des pseudonymes. 
ensemble de valeurs associées (où se rencontrent les intérêts de l'institution et du détenu), fournir des stimulants sous forme de privilèges, imposer le respect par certains droits par le personnel ou maintenir certains contacts extérieurs. » (Mary, 2006, p. 390).

Il convient dès lors de dépasser la seule définition générique que Goffman donne de l'institution totale dès le propos introductif de son ouvrage où celleci est décrite comme « un lieu de résidence et de travail où un grand nombre d'individus, placés dans la même situation, coupés du monde extérieur pour une période relativement longue, mènent ensemble une vie recluse dont les modalités sont explicitement et minutieusement réglées »(Goffman, 1968, p. 41), et de saisir la façon dont l'institution opère sur ses membres une forme d'emprise davantage mentale et symbolique, dont les processus atteignent l'identité personnelle. Une étude ethnographique réalisée sur les écoles de la « dernière chance » en Communauté française de Belgique fournit des pistes utiles dans cette perspective.

L'auteur, Philippe Vienne, effectue un rapprochement pertinent entre ce type de dispositif quant au « sentiment d'enfermement » qu'il procure et l'institution totale, précisément dans « l'exacerbation de l'emprise » qu'elle exerce sur ses membres (Vienne, 2005). Les écoles dont il est question ici revêtent les attributs d'un monde clos alors qu'elles n'ont pas les attributs spatiaux des CEF. En effet, "l'école "diurne" relâche ses élèves en fin de journée vers d'autres socialisations que la sienne [...]. L'absence d'assignation à résidence [...] écarterait donc la possibilité d'avoir recours au concept d'institution totale » (art. cit, p. 66). Pour autant, en reprenant à son compte l'interprétation qu'a pu faire H.S. Becker des travaux de Goffman, l'auteur porte la focale sur une seconde dimension de l'institution totale qui se concentre davantage sur un principe déterminant, «l'emprise » plus ou moins " enveloppante » de l'institution sur ses membres (ibid.). Ainsi, Goffman considérait de son côté que « toute institution accapare une part du temps et des intérêts de ceux qui en font partie et leur procure une sorte d'univers spécifique qui tend à les envelopper. Mais parmi les différentes institutions de nos sociétés occidentales, certaines poussent cette tendance à un degré incomparablement plus contraignant que les autres. [...] Ce sont ces établissements que j'appelle "institutions totalitaires". » (Goffman, 1968, p. 45-46). Ph. Vienne rappelle sur cette base que la notion d'espace « enveloppant » s'inscrit pour Goffman dans une logique de « totalisation », où l'auteur " vise donc ici moins l'absence ou la présence de caractère résidentiel dans l'institution, que la capacité de celle-ci à réaliser une emprise sur ses membres, à les insérer dans un univers spécifique » (art. cit., p. 67). Si ces écoles ne sont pas des institutions totales, elles sont néanmoins traversées par une certaine rationalité totalisante qui s'impose à ses usagers.

Appliqué aux CEF, le concept d'emprise permet de jeter un regard plus mesuré sur l'usage des notions de « fermeture » et de « discipline » qui sont 
spontanément utilisées par la critique courante les visant, provenant notamment des syndicats de personnels éducatifs pour le mineurs et de certains commentateurs.

\section{CONFIGURATIONS SPATIALES ET SYMBOLIQUeS}

L'enquête a en effet révélé une forme d'emprise exercée par l'institution sur les mineurs placés en CEF mais également sur les personnels eux-mêmes. Cela nous autorise à dépasser la seule conception de l'enfermement comme clôture juridique et matérielle, pour saisir les modalités d'emprise symbolique de l'institution dans des espaces d'interaction entre le personnel et les mineurs, à la fois enfermante, contraignante et enveloppante.

\section{Emprise sur le comportement : temps et espace}

Le postulat qui était au fondement de la création des CEF tient dans la propriété éducative présumée de la contenance institutionnelle et comportementale. D'inspiration psychanalytique autant que comportementaliste, la contenance trouve sa traduction dans un encadrement éducatif renforcé où le quotidien des mineurs se trouve fortement occupé, quadrillé et réglé par l'institution. Il est tentant de mobiliser ici la notion de « discipline» mais le projet sous-jacent à cette méthode renvoie davantage à la « responsabilisation » individuelle (Milburn, 2009) qu'à une discipline des corps telle que théorisée par Foucault (1975).

Ainsi, dans le hall d'entrée du CEF de Fromintin, un grand tableau permet d'afficher, chaque jour, le programme d'activité de chaque jeune, rythmé heure par heure. En effet, le temps des jeunes placés en CEF est organisé et structuré autour d'un emploi du temps individuel fait d'activités ordinaires, ludiques, scolaires et professionnelles très cadré. La gestion du temps est posée comme un levier éducatif élémentaire visant à casser un rythme de l'extérieur fait d'oisiveté nocturne, remplacé ici par un surcroît d'activités dès le matin. Mais l'affichage engage à une forme de réflexivité visant à ne pas simplement se plier à l'institution mais s'approprier la valeur des activités et de leur temporalité, et d'y adhérer de façon à intérioriser le sens des normes ainsi prétendument transmises.

Si le mineur est censé s'approprier la signification de cette scansion temporelle pour y trouver son intérêt personnel (d'où l'affichage), il n'en reste pas moins que le cadrage temporel et le contenu des activités prévues dans les tranches sont fixés par l'institution. Le processus d'appropriation des normes dans une dynamique autonome participe de son emprise en dernier ressort, quand bien même il mobilise l'implication des mineurs placés.

Ce cadrage trouve son extension dans l'espace dans la mesure où chaque temps est associé à une zone qui comporte une part de contrainte et de fermeture variable, selon les CEF enquêtés. Les repas et les soirées ont lieu dans des 
espaces confinés que sont la salle à manger et la pièce commune, où se déroule l'essentiel de la sociabilité ordinaire, encadrée par les personnels éducatifs. La cour, la salle ou le terrain de sport, les espaces verts et les ateliers offrent un potentiel de circulation et de mouvement ainsi qu'une possibilité de sociabilité modulée, parfois de mise à distance provisoire des encadrants. Ces espaces recèlent ainsi une dimension intermédiaire comportant un degré de liberté de mouvement et l'accès à des espaces plus ouverts ou renvoyant à la vie extérieure. Enfin, il existe des possibilités de sorties provisoires à l'extérieur du centre, que nous examinons plus loin.

\section{Une emprise sur l'identité individuelle}

Ce processus de socialisation par l'institution comporte un potentiel de dépersonnalisation là où il est censé acquérir une fonction d'autonomisation et de responsabilisation. Dans la mesure où l'institution pose ce cadre que le mineur, qui y est placé, est censé s'approprier, l'effet contraire est tout aussi possible : le mineur met en suspens une autonomie qui reste immature et qu'il associe à sa vie dans son milieu habituel (famille, quartier, amis, etc.) et se moule dans la logique spatio-temporelle de l'institution. Une éducatrice nous confie ainsi qu'elle est plus inquiète pour les mineurs qui se sentent bien au CEF, « dans leurs pantoufles », que pour ceux qui sont en rébellion permanente et résistent à la rationalité institutionnelle imposée par la configuration spatiotemporelle enveloppante. Dans la même perspective, certains mineurs considèrent que la prison constitue une option préférable au CEF dans la mesure où la contrainte y recèle une fonction purement punitive et disciplinaire, et ne comporte pas d'objectif de socialisation, et donc d'emprise sur la formation de leur personnalité.

Dans l'imaginaire des mineurs, seul le comportement durant le séjour est visé par les contraintes carcérales qui ne cherchent pas à intervenir sur la formation de leur personnalité, mais seulement à leur montrer le risque que comportent les actes déviants. Aussi certains d'entre eux n'hésitent pas à commettre des actes induisant un risque d'incarcération (violences, fugue).

\section{Déclinaisons de la fermeture : la symbolique de la clé}

Le processus de dépersonnalisation se manifeste dans la façon dont l'institution contrôle les espaces « privés », en verrouillant systématiquement l'accès aux lieux personnels (chambres des adolescents, sanitaires, etc.), et à ceux $d u$ personnel (bureau du personnel d'encadrement, salle d'art thérapie, local scolaire, bureau de la psychologue...). Le marquage entre monde intérieur et monde extérieur se trouve ici décliné et gradué par la symbolique de la «clé ». Le passage entre ces différents espaces contribue à définir à chaque fois ce qu'ils portent comme valeur symbolique associée aux fonctions de l'institution et à ses 
effets. Le rituel de l'ouverture et de la fermeture des portes auquel se livre continument le personnel confère explicitement aux frontières spatiales leur fonction de surveillance et de gardiennage, attribuant aux mineurs placés un statut de danger ou de menace potentiels. En outre, les « clés » sont souvent en réalité des « badges » qui actionnent des verrous-ventouses électroniques : ceux-ci donnent un caractère plus fluide et anodin au franchissement des frontières en comparaison des trousseaux de clés et des claquements des verrous propres au milieu carcéral. Ainsi les personnels éducatifs peuvent-ils se distinguer des surveillants de prison (Bailleau et al., op. cit.).

Alors qu'ils sont officiellement assignés à un rôle « éducatif », les personnels occupent ainsi bon gré mal gré une fonction privative et de contrôle des corps dans l'espace. Par ce rituel de différentiation des espaces avec cet objetfrontière que constitue la clé, l'institution assoit son emprise sur les éducateurs eux-mêmes, qui incorporent le schéma institutionnel dans leur routine et dans leur mode de fonctionnement dans l'espace. Un rôle qu'ils revendiquent parfois comme nécessaire pour exercer leur compétence pédagogique et qu'ils regrettent à d'autres moments comme une entrave à certains aspects de sa réalisation. Aussi sont-ils contraints de se positionner en toute circonstance par rapport au schéma des frontières institutionnelles. Il semble que se joue ici de façon principalement symbolique, le marquage d'une rupture entre monde extérieur (ou antérieur au CEF), et monde intérieur du CEF, un monde spécifique et fermé, dont la « clé» fonctionne comme métaphore.

\section{Le rituel de dépouillement : espace institutionnel et identité personnelle}

Ainsi, de la même façon que la symbolique de la clé renvoie aux codes des frontières institutionnelles, on peut voir dans la "cérémonie du dépouillement », pour reprendre les termes employés par Goffman dans Asiles, une manière pour le CEF de sceller mentalement la séparation qui doit exister entre l'espace de la puissance institutionnelle du CEF et le monde extérieur, ouvert et libre. Lors de leur arrivée dans les CEF, les mineurs se voient privés de leurs vêtements de " marque », qui constituent un élément majeur d'identité personnelle à l'extérieur, et attribuer des habits quelconques par le centre. S'il ne s'agit pas d'uniformes de reclus, il s'agit bien d'un nivellement institutionnel. Les mineurs se voient également retirer toute une série d'objets personnels qui sont interdits dans les locaux. Le plus notoire, le téléphone mobile, voit son usage interdit, pour des raisons de sécurité mais surtout pour inciter à couper les ponts avec leurs relations à l'extérieur (copains, complices, partenaires en amour mais aussi pourvoyeurs de drogue, etc.). C'est une rude épreuve pour des adolescents chez qui le téléphone représente l'ouverture aux divers espaces sociaux.

Une telle cérémonie de dépouillement contribue à faire de la courte période d'accueil - qui vise à évaluer l'adaptation du mineur à la vie du 
centre et à lui laisser prendre connaissance des principes qui l'animent, autrement dit du schéma institutionnel - un rituel de dégradation, selon les mots de Goffman, et il marque ainsi brutalement la frontière entre l'antérieur-extérieur et l'intérieur-postérieur, sous la forme d'une rupture mentale, symbolique, spatiale, temporelle et institutionnelle qui consacre d'entrée de jeu l'emprise sur chaque nouvel entrant.

\section{Une emprise professionnelle?}

Aussi les rituels du dépouillement, et plus largement de l'accueil ( welcoming ceremony » chez Goffman), agissent-ils avec la même force institutionnelle sur les éducateurs qui la mettent en œuvre. L'emprise s'exerce également sur eux dès lors qu'ils sont amenés à conférer une valeur à cette action dès lors que ces personnels se voient équipés de schémas institutionnels qui s'imposent à eux, notamment en termes de définition du dedans/dehors, que traduit l'activité de (dé)verrouillage. L'équipe exprime ainsi le sentiment d'être collectivement et constamment soumis au schéma mental qu'impose l'institution.

Ainsi, nombre d'entre eux ne quittent pas mentalement le CEF quand ils sortent de son espace à la fin de leur service. L'implication se prolonge lorsqu'ils sont interpellés par leurs collègues pour traiter une question, ou lorsqu'eux-mêmes prennent spontanément contact pour prendre des nouvelles, suite à un problème rencontré durant leur présence.

« Je me demande parfois si je vais y arriver, je n'ai pas forcément envie de me lancer le défi. Je fais en fonction de ce que je suis capable de donner. En tout cas, je suis en train de me poser la question. Faudrait-il que je fonctionne comme mes deux collègues, non-stop, avec 30 SMS par jour ? Est-ce que je suis obligé de me mettre à leur niveau ? C'est ça la question. Ou bien je vais fonctionner différemment» (Chef de service d'un CEF).

«Quand je suis arrivée ici, il y avait une boîte mél Yahoo et je pouvais regarder tout le temps mes méls. Le deuxième objet, c'est le téléphone, le smartphone où je reçois mes méls en direct. Et j'avoue, c'est une addiction. Même pendant les réunions, je suis capable de répondre à mes méls. Mon portable est tout le temps avec moi. Le seul endroit où je ne me permets pas de l'utiliser, c'est quand je suis avec ma Directrice parce que je sais bien qu'elle déteste ça » (Directrice d'un CEF).

Les canaux de communication contemporains tels que le téléphone portable et le mél, de par leur potentiel d'instantanéité de l'information, recèlent dans leur usage, paradoxalement, un effet d'emprise tout aussi puissant que la privation du téléphone chez les mineurs. Là où sa privation isole le mineur de l'extérieur de l'institution, le «portable » relie les éducateurs (et les personnels de direction encore davantage) avec l'intérieur, contribuant, symboliquement et réellement, à ne jamais rompre le lien entre l'équipe et l'institution, voire les mineurs qu'ils ont en charge. Il en résulte parfois un épuisement 
mental qui explique que les personnels éducatifs se renouvellent de façon très régulière. La tension existant dans l'espace confiné est accentuée par ce lien constant qui traduit une forme d'emprise mentale.

Ainsi, au sein du CEF, la fermeture spatiale se double d'une fermeture symbolique qui confère son sens à la frontière entre dedans et dehors, et qui contribue à la décliner et la graduer. La privation de téléphone cellulaire, le contrôle de la communication avec l'extérieur, y compris la famille, le dosage des sorties accompagnées, la gestion par les éducateurs du nombre de cigarettes autorisées au cours de la journée sont autant de réitérations symboliques de la contrainte spatiale, qui ont des conséquences sur le rapport des mineurs à l'espace contenant : volonté de quitter les lieux, violences liées à la contenance spatiale, etc. mais aussi adaptations à la nature du séjour qui dure en principe six mois et réappropriation du monde extérieur au sein du monde fermé qu'est le CEF. Maitrise du temps, rituel de dépouillement à l'entrée dans l'institution et construction des espaces symboliques où s'exerce la contrainte sont autant de dispositifs institutionnels qui procèdent d'une dépersonnalisation au cours du séjour en CEF.

Ceux-ci forment-ils des « institutions totales » comme l'asile psychiatrique analysé par Goffman ou la prison telle que théorisée par Foucault (op. cit.) ? Le fait que des sorties soient organisées, et qu'il existe une fonction d'abord éducative ne permettent sans doute pas de les qualifier comme tels. Toutefois, le fait que le CEF vive dans l'ombre de la prison, qui constitue la destination prévisible de ceux qui ne se soumettent pas au schéma institutionnel, indique que les CEF se situent dans la perspective de celle- $\mathrm{ci}^{6}$. Autrement dit, la totalisation de l'institution, lisible dans ses schématisations spatiales et temporelles (configurations de frontières), représente l'ultima ratio de la fermeture spatiale des centres et de son schéma institutionnel sous-jacent, mais néanmoins omniprésent.

Le caractère non totalisant de l'emprise se traduit par des écarts à la norme, tant de la part des personnels que des pensionnaires. Ces espaces d'ouverture permettent que s'exerce une forme de socialisation et de réappropriation identitaire dans la mesure où des comportements et des relations acquièrent une valeur positive pour les mineurs comme pour les personnels. $\mathrm{Ce}$ processus ne tient pas à la seule liberté ainsi gagnée sur l'institution et ses contraintes, mais acquiert une valeur éducative dans l'interaction entre les mineurs placés et les adultes qui les encadrent.

6. En effet, les fugues et les refus violents d'autorité sont sanctionnés par un dépôt de plainte automatique qui peut conduire le tribunal à prononcer une peine ferme pour irrespect de contrôle judiciaire. Cela n'est pas systématique mais l'invocation de ce risque est permanente dans les CEF. 
Cela combine les pratiques d' « adaptations secondaires » révélées par le travail de Goffman dans Asiles, qui traversent l'institution totale, et l'élaboration d'un système de dons/contre-dons (Benguigui, 1997) entre personnels éducatifs et pensionnaires qui permet de rééquilibrer les effets des schémas institutionnels et contribue à la part d'efficacité pédagogique au sein ou en dépit ce ceux-ci. Ils font l'objet de la seconde partie de notre propos.

\section{ESPACES INTERMÉDIAIRES ET ADAPTATIONS SECONDAIRES}

Dans son travail magistral consacré à l'asile psychiatrique, le propos de Goffman ne visait pas tant la dénonciation de l'emprise institutionnelle (ce qui a pourtant été principalement retenu) que la capacité des « reclus » à échapper à ce destin, à recréer une identité d'acteur indépendamment de celle-ci dans des pratiques déviantes et des espaces intermédiaires. Ainsi, le reclus ne se réduit pas à ce que l'institution lui impose comme statut et comme personnalité (malade mental en l'espèce) mais acquiert une part d'autonomie qui est toutefois limitée à l'espace physique et symbolique qu'il parvient à se frayer au sein de l'institution et en dépit de son emprise. Qu'en est-il dans le cas des CEF étudiés et surtout quant à la manière dont les mineurs s'approprient les équipements institutionnels?

La réponse passe tout d'abord par l'investissement des espaces intermédiaires, ceux qui acquièrent dans les interstices de l'institution, le statut d'« espaces clandestins », pour reprendre le concept de Goffman.

\section{Espaces clandestins et pratiques transgressives}

Chaque CEF recèle des espaces légèrement protégés du regard institutionnel, mi-ouverts (en plein air) mi-fermés (protégés par des murs ou un toit) : des escaliers d'urgence, un abri, un recoin...

Au CEF de la Briquetterie, un abri placé au bout de la cour est occupé durant les temps non cadrés par l'institution : c'est là qu'on fume, qu'on discute entre jeunes, qu'on chahute, qu'on parle des difficultés qu'on rencontre, qu'on prévoit ce qu'on fera de ses sorties. Bien que les jeunes y soient assez visibles, l'abri prévu pour les intempéries fonctionne comme un abri symbolique vis-à-vis de l'emprise institutionnelle et on s'y réunit quelle que soit la météorologie.

Tous les CEF observés comportent des endroits semi-clandestins de ce type, où les mineurs se réfugient durant les plages temporelles non contrôlées (après les activités, avant le repas, les week-ends, etc.). Ils représentent un moment de liberté symbolique et temporaire qui fonctionne comme une soupape de sécurité. Les éducateurs y sont parfois admis dans la mesure où ils y quittent leur habit institutionnel et se positionnent dans une proximité : ils n'imposent pas des normes mais sont à l'écoute et interviennent sur le mode de l'échange 
et de la parité. Ce sont des espaces de défoulement, où chaque mineur peut librement exprimer sa personnalité : personnage public bravache ou personne intime plus fragile.

Les espaces verts constituent un défouloir type : censés apprendre des éléments de savoir horticole, les engins sont détournés pour faire une course de tondeuse ou simuler un combat héroïque contre les arbustes avec une débrousailleuse. Les salles communes jouent également ce rôle quand la météo ne permet pas la présence à l'extérieur, et sont provisoirement démises de leurs propriétés institutionnelles.

Aussi ces espaces clandestins participent-ils des "adaptations secondaires » (pour emprunter ici encore à Goffman) largement tolérées par les éducateurs, qui se positionnent de la sorte à l'interface entre l'emprise institutionnelle et l'autonomie idéalisée. Mieux, ils l'utilisent comme levier éducatif, dans une approche qui vient en contrepoint de leur rôle de gardiennage dans lequel l'institution les positionne. Un processus équivalent a été observé par A. Chauvenet en milieu carcéral adulte : « Afin de gagner la coopération des détenus, d'obtenir leur consentement à leur propre assujettissement, et en même temps pour survivre et éviter les explosions, les surveillants initient un système d'échange [...] fondé sur le don et le contre-don. Ce système d'échanges est initié dans les silences, les marges, les failles de la loi et contre elle. Il est de nature tout à fait informelle bien que toléré parce qu'indispensable » (Chauvenet, 1998, cité par Chantraine, op. cit., p. 305).

«-Et donc sur quoi vous appuyez-vous justement pour construire cette compétence à intervenir dans un cadre contraint?

- Ben déjà, il y a le règlement. Le règlement intérieur du CEF, c'est déjà le cadre. Donc le cadre, normalement, on est tous censés le tenir. Alors là après, moi je mets un bémol, je maintiens le cadre, par contre, il y a des moments précis où il faut savoir... Ben, c'est savoir faire une petite entorse, mais bien expliquer au gamin. Par exemple, j'sais pas, il y a un décès dans une famille. Le gamin il est touché, c'est logique. Le gamin il va partir en vrille un peu dans tous les sens. Quelque part, des fois, il y a un gamin, il veut une cigarette pour se calmer. C'est une transgression, j'ai pas à donner une cigarette, je le sais. Par contre, si on explique au gamin que c'est un truc de type exceptionnel, que c'est plus entre guillemets "thérapeutique" qu'autre chose, il peut très bien l'entendre, sachant qu'il va pas se caler dans la tête que ça devient systématique, je dirais, je fais une intervention ponctuelle sur un problème à un moment donné, sans valider le truc comme étant une... Alors après, oui, c'est hors du cadre, d'accord » (Éducateur technique d'un CEF).

\section{Don et contre-don : de l'usage professionnel des adaptations secondaires}

La logique de don/contre-don prend en CEF une dimension supplémentaire : il s'agit certes d'obtenir la coopération des mineurs pour acquérir une légitimité pour l'action éducative mais au-delà du « consentement à l'assujettissement », 
d'acquérir un accès à leur subjectivité pour ensuite la mobiliser dans le but de réinvestir leur identité personnelle en vue d'une socialisation préparatoire à la sortie. Elle se traduit dans de multiples registres relationnels avec les mineurs, quand la transgression à la règle est suffisamment légère pour que cela ne porte pas à conséquence. Le comptage des cigarettes quotidiennes autorisées apparaît comme le plus emblématique de ce processus qui consiste à échanger un peu de tolérance contre un peu de bienveillance et d'acceptation de l'intervention éducative. Le don/contre-don fonctionne dès lors comme une condition de possibilité de celle-ci et les espaces intermédiaires en représentent l'antichambre.

La salle de classe est typiquement un lieu doté de cette vertu, où les enseignants et les éducateurs qui les secondent parfois obtiennent de la sorte un minimum de participation. Il s'agit ici de convertir un lieu - l'école - à haute tension institutionnelle en lieu à faible tension, où l'institution s'efface légèrement face aux contraintes de la clôture judiciaire. En effet, la plupart des mineurs placés sont totalement ou partiellement déscolarisés depuis longtemps, ce qui place la scolarisation au sein du CEF devant le double défi de redonner un peu de goût à l'apprentissage et d'entreprendre de remettre à niveau des élèves dont les compétences scolaires sont souvent dramatiquement insuffisantes. À cette fin, les enseignants peuvent jouer sur ce qu'ils représentent comme représentatif de l'extérieur du CEF pour faire de l'école un symbole d'émancipation et avoir recours à des pédagogies peu contraignantes (ludiques, etc.) pour faire progresser scolairement ceux qui deviennent alors des élèves. $\mathrm{Ce}$ statut peut de la sorte acquérir un surcroît de valeur, dans la mesure où il est associé au dehors : l'espace de la salle reflète donc cette part d'évasion mentale.

Les moments de sortie sont encore davantage chargés de cette possibilité de réappropriation des adaptations secondaires par les personnels éducatifs. La fonction institutionnelle des sorties consiste à conférer un sens positif à l'exercice d'une liberté, qui s'acquerrait donc progressivement. Il s'agit ici encore d'une pédagogie de la responsabilisation (Milburn, op. cit.) qui s'appuie ici sur la modulation de la contrainte spatiale. Deux types de sortie à caractère éducatif prévalent en CEF : les sorties collectives encadrées et les stages dans le cadre des diplômes scolaires. Il s'agit dans le premier cas d'activités sportives, de visites ou de randonnées visant à sortir du cadre spatial du centre tout en étant encadré par les personnels éducatifs. Les mineurs placés en stage assurent une activité professionnalisante chez un employeur à proximité du centre, qu'ils réintègrent le soir et les autres jours. Les sorties sont autorisées à partir du second bimestre du placement, les stages à compter du troisième. Les mineurs sont également parfois autorisés par le juge à passer un week-end chez leurs parents, vers la fin du séjour.

Une telle progressivité dans l'attribution de l'espace de liberté a un effet sur les mineurs placés: celui d'une idéalisation de l'extérieur du CEF. La 
contrainte de l'espace fermé et des activités contrôlées est souvent vécu de manière négative et la liberté associée aux sorties et donc à l'espace extérieur est attendu avec impatience. Le stage, qui vient comme une promesse après une progression positive, fait l'objet le plus souvent d'une forte implication de la part de ceux qui en bénéficient, d'après les professionnels des CEF, et donne lieu à des expériences favorables du point de vue éducatif dans une grande proportion de cas. On peut dès lors y voir une adaptation primaire aux objectifs de l'institution, ceux d'une préparation à la sortie définitive, par la réussite scolaire entre autres.

La réalité est plus nuancée. La sortie définitive, construite comme projet dans la démarche éducative tout au long du placement de six mois, comporte une forte ambivalence pour les mineurs placés, à la fois espérée comme libération de la contrainte spatiale et comportementale et parfois redoutée parce que c'est un retour au contexte délétère du milieu «naturel», pour reprendre le lexique de l'action sociale. En cela, profiter du stage constitue une forme d'adaptation secondaire, et l'espace extérieur où il se déroule un espace intermédiaire : elle permet de s'extirper de l'emprise du CEF, de ses contraintes et de la vie collective entre mineurs placés, et d'avoir des contacts avec d'autres personnes.

La tension entre cet extérieur provisoire et intermédiaire et la sortie effective apparaît dans des situations de mise en échec volontaire de la part d'une proportion non négligeable de mineurs, partagés entre le désir de liberté et la crainte du milieu d'origine. Malgré un projet professionnel investi de façon réussie, ils le mettent en échec à quelques jours de leur sortie du CEF. Cela consiste à commettre un acte délictueux au sein du CEF (violence, fugue, etc.), voire chez l'employeur (vol dans la caisse, esclandre, casse...). La " sortie » réelle s'approchant, elle acquiert progressivement une dimension anxiogène : il va falloir quitter l'espace enveloppant du CEF qui, s'il a un effet contenant et d'emprise institutionnelle, n'en acquiert pas moins, au fil du temps, une dimension de protection. Cela est tel que, dans l'un des CEF enquêtés, un mineur qui était de retour dans sa famille après un stage qui s'était très bien passé, s'est donné la mort en se défenestrant, à quelques jours de sa sortie définitive. Heureusement rarissime, un tel geste constitue la forme hyperbolique de la tension entre espace extérieur au CEF et réalité de la « sortie », c'est-à-dire du retour à un « extérieur » menaçant.

Un tel acte reflète le hiatus existant entre la sortie projetée et la sortie réelle. En effet, celle-ci ne correspond pas à l'expérience de l'apprentissage, qui trouve toute sa valeur dans le contrepoint avec l'espace fermé, mais elle se traduit par la confrontation avec le revers de la médaille de l'autonomie et de la liberté : famille, obligations du quotidien, ambiance du quartier, échecs de l'école, pression à la consommation... L'enfermement vient idéaliser la liberté, mais n'assure pas pour autant les clés pour l'affronter. La scolarisation et la formation professionnelle sont certainement des instruments d'insertion utiles mais 
dans bien des cas, ils ne suffiront pas à reconstruire une identité malmenée par l'institution et le parcours antérieur à celle-ci.

\section{CONCLUSION \\ ESPACE CONTRAINT ET PROJET ÉDUCATIF : QUELLE COHÉRENCE ?}

Il ressort des éléments recueillis dans nos travaux une forte ambivalence entre les degrés de contraintes imposés aux différents espaces des CEF et leur ambition éducative, fondée sur une responsabilisation davantage qu'une discipline. L'institution totale présente ici ne se traduit pas par un enfermement pur et par une aliénation des « reclus » mais par un jeu sur leurs rares espaces de liberté sur lesquels l'éducatif est amené à s'appuyer pour en jouer en les élargissant progressivement jusqu'à la sortie, qui constitue l'objectif affirmé. Cette emprise très forte implique des espaces intermédiaires de liberté provisoire et tolérée permettant des adaptations secondaires, détournement des équipements institutionnels de leur fonction.

Ce n'est pas le moindre des paradoxes que de constater que les éducateurs doivent se situer dans ces espaces clandestins et ces pratiques quasi-transgressives qui accompagnent les adaptations secondaires. L'emprise institutionnelle, voulue par les promoteurs des CEF comme élément majeur de la dynamique éducative, n'a d'effet que par défaut et dans les ambivalences qu'elle induit. Les espaces contraints et leur symbolique ne fonctionnent pas comme cadres à la responsabilisation (socialisation à la norme dominante) mais comme contrepoint pour l'action éducative, qui doit mobiliser un idéal de l'extérieur et des quasi-transgressions comme leviers.

\section{RÉFÉRENCES BIBLIOGRAPHIQUES}

Bailleau, F. ; Gourmelon, N. ; Milburn, Ph. 2012. Les établissements privatifs de liberté pour mineurs : entre logiques institutionnelles et professionnelles. Une comparaison entre EPM, QM et CEF, Rapport de recherche pour la Mission de recherche Droit et Justice, Cesdip-CNRS ; Printemps-CNRS.

Benguigui, G. 1997. «Contrainte, négociation et don en prison », Sociologie du travail, $\mathrm{n}^{\circ} 39-1$, p. 1-17.

Bourdieu, P. ; Passeron, J.-C. 1970. La Reproduction, Paris, Minuit.

Chantraine, G. 2000. «La sociologie carcérale : approches et débats théoriques en France ». Déviance et Société n 3, Vol. 24, p. 297-318.

Chauvel, L. 2006. Les classes moyennes à la dérive, Paris, Seuil.

CHAUVENET, A. 1998. « Guerre et paix en prison », Les cahiers de la sécurité intérieure $\mathrm{n}^{\circ} 31$, p. $91-100$.

Combessie, Ph. 2000. "Ouverture des prisons... Jusqu'à quel point?", dans D. Lhuilier (sous la dir. de), La prison en changement, Toulouse, érès, p. 69-99.

DuBAR, C. 2002. La socialisation, construction des identités sociales et professionnelles, Paris, Armand Colin. 
Dubar, C. 2001. La crise des identités, Paris, Puf.

FoucAult, M. 1975. Surveiller et punir. Naissance de la prison, Paris, Gallimard.

Goffman, E. 1968. Asiles. Études sur la condition sociale des malades mentaux, Paris, Minuit, coll. « Le sens commun ».

Goffman, E. 1975. Stigmate. Les usages sociaux des handicaps, Paris, Minuit, coll. « Le sens commun ».

MARY, Ph. 2006. «La prison en Belgique : de l'institution totale aux droits des détenus ? ", Déviance et Société n ${ }^{\circ} 3$, Vol. 30, p. 389-404.

Milburn, Ph. 2009. Quelle justice pour les mineurs? Entre enfance menacée et adolescence menaçante, Toulouse, érès.

Rostaing, C. 1997. La relation carcérale. Identités et rapports sociaux dans les prisons de femmes, Paris, Puf.

VIENNE, Ph. 2005. « De l'institution totale à l'institution scolaire. La grille de lecture goffmanienne d'une ethnographie scolaire dans l'enseignement professionnel », La matière et l'esprit $\mathrm{n}^{\circ} 2$, p. 63-81. 